\title{
Toward a Formalization of FrameNet in Situation Theory
}

\author{
Cenk Atlig, Yılmaz Kılıçaslan, and Gurkan Tuna
}

\begin{abstract}
FrameNet is a semi-formal lexical database. There are various attempts to formalize this database. This paper presents a partial formalization of FrameNet in situation theory. This mathematical theory of information may provide a fruitful basis for making FrameNet accessible to other fields such as natural language processing, computational linguistics and informational retrieval.
\end{abstract}

Index Terms-FrameNet, frame elements, frame-to-frame relations, situation theory.

\section{INTRODUCTION}

The aim of this paper is to explore the ways of translating FrameNet into situation theory to some extent. FrameNet is a lexical database, which lacks a formal characterization. We argue and try to demonstrate that situation theory, as a mathematical theory of information, provides a sound and fruitful basis for the formalization of lexical databases like FrameNet.

The paper is organized as follows. Section 2 and 3 provide background information on FrameNet and situation theory, respectively. Section 4 offers some suggestions as to how to translate a fragment of FrameNet into situation-theoretic objects. We conclude in Section 5 with a summary of the paper.

\section{FRAMENET}

FrameNet is based on Frame Semantics, which is a theory of meaning developed by Charles J. Fillmore and colleagues (cf. [1]-[11]. The basic idea behind Frame Semantics is that words in human language are never interpreted in isolation but are always understood with respect to (semantic) frames. ${ }^{1}$ According to Fillmore, a frame is a schematisation of experience (i.e. a knowledge structure) that relates the elements and entities with a culturally embedded scene from human experience. To put it more explicitly, a frame is a description of a situation or entity along with its participants.

A frame is associated with a name and an informal definition in the FrameNet database. For example, the frame representing the meaning of $c u t$ is named Cutting and defined as follows.

1) Informal Definition of the Cutting Frame:

Manuscript received April 6, 2016; revised August 24, 2016.

C. Atlig and G. Tuna are with the Department of Computer Programming, Trakya University, Edirne, Turkey (e-mail: cenkatlig@trakya.edu.tr, gurkantuna@trakya.edu.tr).

Y. Kılıçaslan is with the Department of Computer Engineering, Trakya University, Edirne, Turkey (e-mail: yilmazkilicaslan@trakya.edu.tr).

${ }^{1}$ Ref. [12], too, presents a persuasive argument in favor of the view that words cannot exist in one's mind as in a dictionary, but using the term 'domain' instead of 'frame'.
An Agent cuts an Item into Pieces using an Instrument (which may or may not be expressed).

Each frame comes with a number of core and non-core frame elements which can be thought of as the semantic roles played by the participants. Core frame elements are those which are "necessary to the central meaning of the frame" [13, p. 133]. Below are the core frame elements specified for the Cutting frame.

2) Core Frame Elements of the Cutting Frame:

Agent The Agent is the person cutting the Item into Pieces.

Item The item which is being cut into Pieces.

Pieces The Pieces are the parts of the original Item which are the result of the slicing.

Non-core frame elements are those that are not necessary for a unique characterization of the frame. Here are the non-core frame elements specified for the frame Cutting.

3) Non-Core Frame Elements of the Cutting Frame:

Instrument The Instrument with which the Item is being cut into Pieces.

Manner Manner in which the Item is being cut into Pieces.

Means An act of the Agent that accomplishes the slicing.

Place Where the slicing takes place.

Purpose The purpose for which the Item is being sliced into Pieces.

Result The Result of the Item being sliced into Pieces.

Time When the slicing occurs.

The description of a frame in the FrameNet database contains also the lexical units (corresponding word senses) that evoke the frame. (4) shows the list of lexical units that the Cutting frame includes.

4) Lexical Units of the Cutting Frame:

carve, chop, cube, cut, dice, fillet, mince, pare, slice

FrameNet additionally includes a network of relations between frames. Below are four of them.

5) Four Frame-to-Frame Relations:

Inheritance Frame A inherits from frame B, if $\mathrm{A}$ is a

Using Frame A uses frame B, if A presupposes $\mathrm{B}$ as background. 
Subframe

Frame A is a subframe of frame $\mathrm{B}$, if $\mathrm{B}$ is a complex frame containing $\mathrm{A}$ as a part.

Precedes The precedes relation captures a temporal order that holds between subframes of a complex scenario.

The only frame-to-frame relation specified in the FrameNet database for Cutting is that of Inheritance. The specification indicates that Cutting inherits from the frame Intentionally_affect. As for this latter frame, it inherits from two other frames, namely Intentionally_act and Transtive_action. FrameNet has developed a visualization tool, FrameGrapher, for viewing the relations between frames and their frame elements. Fig. 1 shows the diagram that FrameGrapher generates in response to a request for viewing the Inheritance relations starting from the Cutting frame.

The frame Intentionally_affect is associated with the following definition:

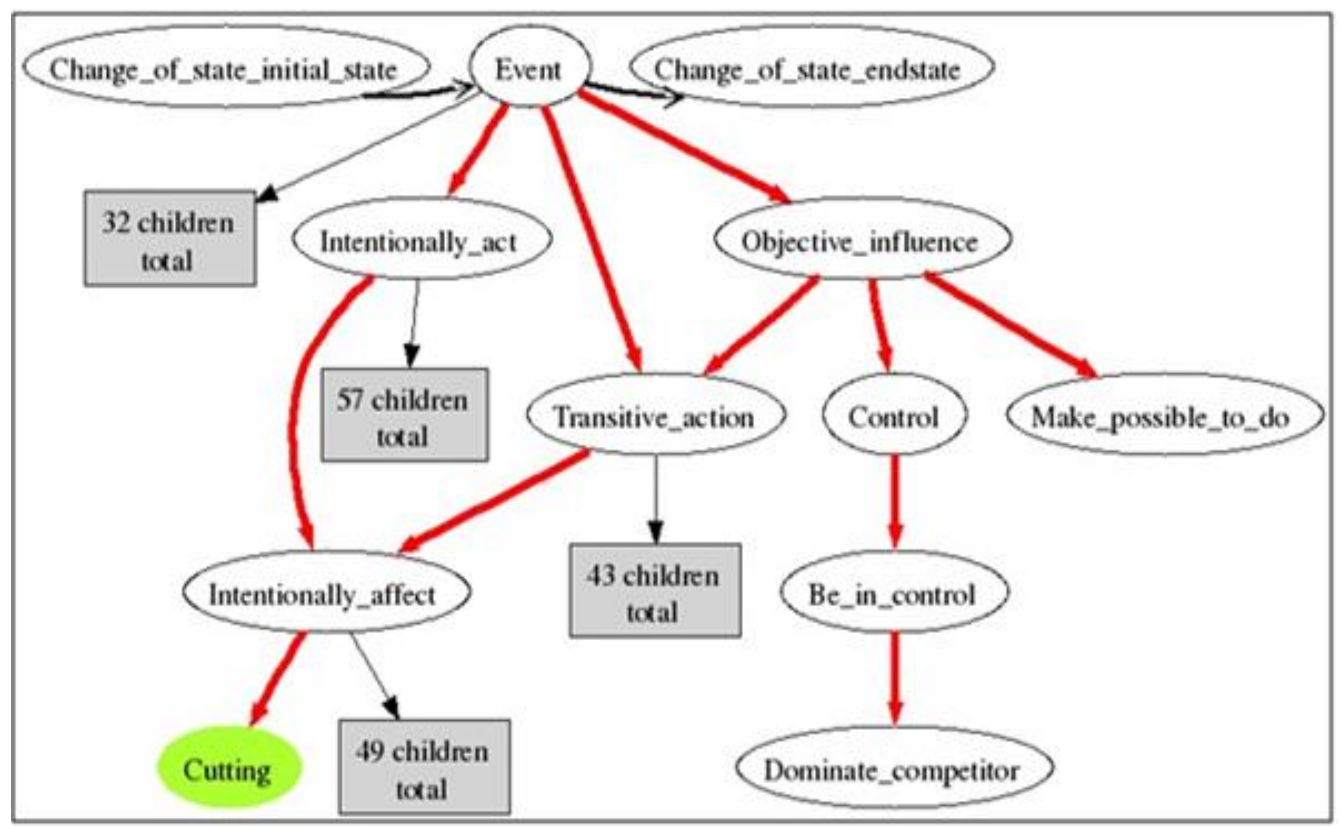

Fig. 1. A fragment of the Inheritance relations starting from the frame Cutting.

6) Informal Definition of the Intentionally-Affect Frame:

An Agent causes a Patient to be affected, sometimes by a particular Means or by use of an Instrument.

Agent and Patient are specified in the FrameNet database as the core frame elements of this frame:

7) Core Frame Elements of the Intentionally-Affect Frame:

Agent The conscious entity, generally a person, that performs the intentional act that affects the patient.

Patient Patient is the entity acted on and that may, but need not, undergo a change.

In order to go into a situation-theoretic formalization of FrameNet, we first need to introduce some background on situation theory. This we will do in the next section.

\section{SITUATION THEORY}

Situation theory ${ }^{2}$ is a mathematical theory of information. What is central to this theory and what gives rise to its name is the treatment of information as something about some situation. Situations are limited parts of reality and identified

${ }^{2}$ See [14] and [15] for an original version of situation theory introduced in the early $80 \mathrm{~s}$, and [16] for a survey of changes and developments that have taken place afterwards. by the basic items of information they support. Such items of information are called 'infons".

Each basic infon has a relation and a number of arguments and is either positive or negative. The general form of a (basic) infon is sometimes represented in linear notation as follows: ${ }^{4}$

8) $<<$ Rel, $\operatorname{Arg}_{1}, \ldots, \operatorname{Arg}_{n} ;$ Pol $>>$

where Rel is an n-place relation; $\arg _{1}, \ldots, \arg _{n}$ are objects appropriate for Rel; and Pol is the polarity, which might be either 0 or $1 .^{5}$ For instance, ignoring temporality, the infon corresponding to 'John kicked Fido' can be written as:

9) $<<$ kick, j, f, $1>>$

where $\mathrm{j}$ and $\mathrm{f}$ stand for the individuals called 'John' and 'Fido', respectively.

Given any two infons, say $\sigma$ and $\tau$, we can construct two compound infons via conjunction:

10) $\sigma \wedge \tau$

${ }^{3}$ The invented word "infon" was introduced by Keith Devlin in the late 1980s. Infons are also referred to as states of affairs, soas, or (possible) facts in the literature.

${ }^{4}$ See [17]

${ }^{5}$ A notation like (8) only encodes the valence for any given relation. As each relation comes with a set of argument roles, a richer notation, like (i), is sometimes used to specify these roles (cf. Gavron and Peters 1990): 
and disjunction:

11) $\sigma \vee \tau$.

In situation theory, a piece of information is seen as different from a proposition. Infons are not things that in themselves are true or false. They may hold or not relative to a situation. As for propositions, they are true or false on their own. They require not just a piece of information but also a situation against which to evaluate it. Given a situation, $s$, and an infon, $\sigma$, we can construct a proposition, denoted as:

12) $\mathrm{s} \vDash \sigma$.

This is read as "s supports $\sigma$. "

In some formulations of situation theory, objects can have restrictions placed upon them. Only propositions can restrict an object. [18], an "illative" approach to logic, treats restrictions by means of a connective $\mathrm{I}$. If $\mathrm{B}$ is an object and $\mathrm{P}$ is a proposition, we can pair $\mathrm{B}$ with $\mathrm{P}$ to yield a restricted object, represented as below.

(i) $<<$ Rel, $_{\text {Role }}$ : $\operatorname{Arg}_{1}, \ldots$, Role $_{n}$ : $\operatorname{Arg}_{n}$; Pol $>$.

13) $\mathrm{B} \uparrow \mathrm{P}$.

If $\mathrm{P}$ is true then the restricted object is identical to its unrestricted counterpart; otherwise the expression ' $\mathrm{B} \uparrow \mathrm{P}$ ' is not even well-formed. To illustrate, consider once again the sentence 'John kicked Fido'. What is foregrounded in this sentence is that the individual named 'John' kicked the individual named 'Fido', not that the kicker is named 'John' and the kickee is named 'Fido'. The latter piece of information serves as background information restricting the foregrounded part. This informational partition can be shown as follows:

$$
\begin{aligned}
14)<<\text { kick, j, f, } 1>> & <<\text { named, j, 'John' }>> \\
\wedge & <<\text { named, f, 'Fido' }>>\text {. }
\end{aligned}
$$

Another important point to note about situation theoretic objects is that any of their constituents can be replaced by a parameter. A parametric object obtained in this way can be thought of as a generalization over its non-parametric counterpart. When a parameter is assigned a value, we get a particular instance of the parametric object.

Another operation that can be applied to parameters is that of abstraction. The notion of abstraction in situation theory is based on work in [19] and [20]. In general terms, it is an operation by means of which a parameter of a parametric object is effectively removed, leaving behind only its 'slot'. In that sense, it is not different from the notion of abstraction used in the $\lambda$-calculus. However, there is a crucial difference between abstraction in the $\lambda$-calculus and that in situation theory (i.e. Aczel-Lunnon abstraction). While in the former only serial abstraction is available (i.e. at each time only one variable can be abstracted over), in Aczel-Lunnon abstraction, several parameters may be abstracted over simultaneously, as well as serially. In case of simultaneous abstraction the roles of the resulting abstract need to be indexed with some role indices. To illustrate, given a parameteric object, $\phi$, with the parameters $\mathrm{X}$ and $\mathrm{Y}$ being its constituents, ${ }^{6}$ we can have an abstract denoted as follows.

\section{5) $\left[\right.$ Role $_{1}: X$, Role $\left._{2}: \operatorname{Arg}_{1} \mid \phi\right]$.}

The order in which the parameters and their role indices is written in the place to the left of the symbol | is to be taken as not significant.

One of the goals of situation theory is the development of a "situated logic". This is a logic based upon on information content rather than upon truth conditions, as is the case with "classical logic." It is through "constraints' that the way that agents make inferences and act in a rational fashion is captured in situation theory. Constraints are linkages between situation types. A situation-type is obtained by abstracting the situation parameter in a proposition like (11):

16) $[S \mid S \vDash \sigma]$.

Suppose that there is a constraint linking (14) to the situation type below.

17) $[S \mid S \vDash \tau]$.

This is denoted by:

18) $[S \mid S \vDash \sigma] \Rightarrow[S \mid S \vDash \tau]$

And serves to infer that if there is a situation of type (16) then there is also a situation of type (17).

Admittedly, the explanations above can be a little difficult to digest without concrete examples. Hopefully, our attempt to partially translate FrameNet into situation theory will also serve to exemplify what is said so far about this theory.

\section{FROM FRAMENET TO SITUATION THEORY}

Having equipped with the situation theoretic machinery introduced in the previous section, we can give a partial formulation of FrameNet in situation- theoretic terms. We will illustrate the formulations we propose with the Cutting frame.

\section{A. Core Frame Elements}

Let us begin with core frame elements. It seems natural to take the following infon as an essential component of the frame Cutting.

\section{9) $<<$ cutting, X, Y, Z; $1>>$}

However, this cannot be the whole story. Thinking with situation-theoretic notions, we should assume that when a lexical unit (e.g. carve, chop, cube, cut, etc.) evokes this frame in an utterance, it will also introduce into the discourse a parameter that stands for the situation supporting the infon above. This assumption is also in line with the Fillmorean view that a frame is a description of a situation with its participants. Thus, the following parametric proposition comes closer to our frame.

${ }^{6}$ Parameters will be denoted below by capital letters, such as $\mathrm{X}, \mathrm{Y}, \mathrm{Z}$, etc. 
20) $\mathrm{S} \vDash<<$ cutting, $\mathrm{X}, \mathrm{Y}, \mathrm{Z} ; 1>>$

Yet, this object falls short of providing all the information pertaining to the core frame elements of Cutting. It only indicates the number of core elements but does not say anything explicit about the elements themselves. In order to make this information explicit, we propose to use the following abstract.

21) [agent:X, item: $Y$, pieces: $Z \mid S \vDash<<$ cutting, $\mathrm{X}, \mathrm{Y}, \mathrm{Z} ; 1>$

Note that the roles of the abstracted parameters are indexed by the core frame elements. This will allow a parser to correctly link the arguments of a sentence headed by a lexical unit of the Cutting frame to the parameters of that situation-theoretic object.

\section{B. Non-Core Frame Elements}

Two points need to be taken into consideration when formalizing non-core frame elements. First, while the core elements of a frame correspond to the arguments of a lexical unit evoking that frame, the non-core elements correspond to adjuncts or modifiers. Second, the core elements are foregrounded in terms of the information they encode while the non-core elements are more backgrounded in that sense.

In order to capture the argument-adjunct distinction in our formalizations, we take a Davidsonian approach to the argument structure of relations. In the Event Semantics suggested in [21], it is noted that adding a modifier to an $\mathrm{n}$-place predicate should not be treated by forming an $\mathrm{n}+1$-place predicate. In addition to several other logical and semantic problems with such a treatment, Davidson also argues that it is undesirable to have an infinite number of versions of the same predicate in store. Davidson solution to the problem involves introducing an event variable with every predicate and representing the modifiers as simple first-order predicates over the event variable. For instance, the analysis for the sentence in (22) will be as in (23). ${ }^{7}$

22) Jones buttered the toast with a knife in the bathroom.

23) $\mathrm{B}(\mathrm{j}, \mathrm{t}, \mathrm{e}) \wedge \mathrm{WITH}(\mathrm{k}, \mathrm{e}) \wedge \mathrm{IN}(\mathrm{b}, \mathrm{e})$

(B for butter, $\mathrm{j}$ for Jones, $t$ for toast, $\mathrm{e}$ for the event variable, $\mathrm{k}$ for knife, $\mathrm{b}$ for bathroom)

In a similar spirit, we propose to treat each non-core frame element as a parametric infon, where the relation is a 1-place relation (i.e. a property) indexed with the name of the element and the argument is an instance parameter. Further, we take all the infons pertaining to the non-core elements to be supported by the situation that the frame describes. Thus, we have the following parametric proposition for the non-core frame elements of Cutting:

24) $\mathrm{S} \vDash<<$ instrument, I; $1>>\wedge<<$ manner, M; $1>>$

$\wedge<<$ means, W; $1>>\wedge<<$ place, L; $1>>$

$\wedge<<$ purpose, W; $1>>\wedge<<$ result, R; $1>>$ $\wedge<<$ time, $\mathrm{T} ; 1>>$

As for the backgrounded status of the non-core frame elements we suggest that the whole proposition above can be placed as a restriction on the proposition in (20) so as to reflect the difference in their salience:

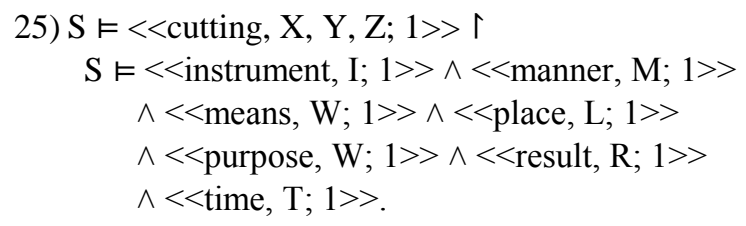

We do not wish to delve into the internal structure of frames any further and we wish to go into the formalization of frame-to-frame relations to a little extent.

\section{Frame-to-Frame Relations}

We propose situation-theoretic treatments for the following frame-to-frame relations: Inheritance, Using, Subframe and Precedes. Let us go over them one by one.

Recall that frame A is said to inherit from B, if A is a subtype of B. This amounts to saying in situation-theoretic terms that A is linked to B via a constraint. In order to specify that constraint, we need to form situation-types uniquely characterizing the frames linked. The foregrounded part of a proposition like (25) is sufficient for a unique characterization of the relevant frame. Therefore, we suggest to specify the constraint between, say, the Cutting frame and the Intentionally_affect frame as in (26).

26) $[S \mid S \vDash<<$ cutting, $X, Y, Z ; 1>>] \Rightarrow$ $\left[\mathrm{S}^{\prime} \mid \mathrm{S}^{\prime} \vDash<<<\right.$ intentionally_affect, $\left.\left.\mathrm{X}, \mathrm{Y}, \mathrm{Z} ; 1\right\rangle>\right]$

The frame Intentionally_affect has three core elements: Agent, Patient and Event.

Recall also that Frame A uses frame B, if A presupposes B as background. Assuming that $\mathrm{A}$ translates into a situation-theoretic proposition $\phi$ and B translates into $\psi$, we propose the following straightforward formulation for the Using relation between these two frames:

\section{7) $\phi \uparrow \psi$}

The Subframe relation corresponds to the part-of relation in situation theory: a situation $\mathrm{s}$ is taken to be part of a situation $s^{\prime}$ if and only if all the infons supported by $s$ are also supported by $s^{\prime}$. Speaking more formally:

28) $s \unlhd s^{\prime}$ if and only if $\{\sigma \mid s \vDash \sigma\} \subseteq\left\{\sigma \mid s^{\prime} \vDash \sigma\right\}$

where $\unlhd$ is the part-of relation between situations.

Lastly, the precedes relation, which captures a temporal order that holds between subframes of a complex scenario, can be easily formalized in situation-theoretic terms as a precedence relation between the time parameters of two situations.

The FrameNet lexical database does not specify the Using, Subframe and Precedes relation of the frame Cutting. 


\section{ADVANTAGES OF FORMALIZING FRAMENET}

As [23] points out, "[o]bscure and intuition-bound notions can neither lead to absurd conclusions nor provide new and correct ones, and hence they fail to be useful in two important respects" (p. 5). According to him, the remedy for such inadequacy is to construct formal models of the subjects under study. From this point of view, we have proposed to formalize FrameNet in situation-theoretic-terms and we have partially shown how to do this in the previous section.

Leaving aside its theoretical benefits, the formalization of a database provides some practical advantages. To begin with, the information that it carries can be encoded in a programming language more easily. In the case of our situation-theroretically formalized FrameNet, this can be done almost in a mechanical way, if the programming paradigm is chosen to be a logic programming paradigm such as Prolog. For instance, the information encoded in (26) can be translated into Prolog as follows:

\section{9) Intentionally_affect(X,Y,Z):- cutting(X,Y,Z).}

As the supporting situations are abstracted away, we leave them unspecified and the coding operation is reduced to a simple reformulation of the relation between the infons. Once FrameNet is transferred into Prolog, we can benefit all advantages of logic programming. We can check the internal structure of a frame, or a frame-to-frame relation with a single query. We can automatically deduce all the information encoded. We can automatically detect inconsistences and deficiencies. It is also worth noting that such an implementation will allow for integration with many natural language processing and information retrieval applications and hence will enable them access the information carried by FrameNet.

\section{CONCLUSION}

Having provided some preliminaries about FrameNet and situation theory, we have made suggestions as to how to formalize a fragment of the former in terms of the mathematical objects developed in the latter. We have confined our examination to (core and non-core) frame elements and four of the frame-to-frame relations. The completion of this work will, we believe, offer opportunities and avenues for the fields of natural language processing, computational linguistics, informational retrieval etc.

\section{REFERENCES}

[1] C. J. Fillmore, "Frame semantics and the nature of language," in Origins and Evolution of Language and Speech, S. R. Harnad, H. D. Steklis and J. Lancaster, Eds. Annals of the NY Academy of Sciences, 1976a, vol. 280.

[2] C. J. Fillmore, "The need for frame semantics within linguistics," Statistical Methods in Linguistics, vol. 12, pp. 5-29, 1976 b.

[3] C. J. Fillmore, "Scenes-and-frames semantics," in Linguistics Structures Processing, A. Zampolli, Ed. Amsterdam and New York: North Holland Publishing Company, 1977a, pp. 55-81.

[4] C. J. Fillmore, "Frame semantics," in Linguistics in the Morning Calm, Hanshin Publishing, Seoul, 1982, pp. 111-137.

[5] C. J. Fillmore, "Frames and the semantics of understanding," Quaderni di Semantica, vol. 6, no. 2, pp. 222-254, 1985.

[6] C. J. Fillmore, "Valency and semantic roles: The concept of deep structure case," in Dependenz und Valenz / Dependency and Valency,
V. Ágel, L. M. Eichinger, H. W. Eroms, P. Hellwig, H. J. Heringer, and H. Lobin, Eds. de Gruyter. Berlin, 2003, vol. 1, pp. 457-475.

[7] C. J. Fillmore and B. T. S. Atkins, "Toward a frame-based lexicon: The semantics of RISK and its neighbors," in Frames, Fields, and Contrasts, A. Lehrer and E. F. Kittay, Eds., NJ: Lawrence Erlbaum Associates Hillsdale, 1992, pp. 74-102.

[8] C. J. Fillmore and B. T. S. Atkins, "FrameNet and lexicographic relevance," Proceedings of LREC 1998, Granada, Spain, 1998.

[9] C. J. Fillmore and C. F. Baker, "Frame semantics for text understanding," in Proc, WordNet and Other Lexical Resources Workshop, 2001.

[10] C. J. Fillmore and C. Baker, "A frames approach to semantic analysis," in The Oxford Handbook of Linguistic Analysis, B. Heine and H. Narrog, Eds. Oxford: Oxford University Press, 2010, pp. 313-340.

[11] C. J. Fillmore, C. R. Johnson, and M. R. L. Petruck, "Background to FrameNet," International Journal of Lexicography, vol. 16, no. 3, pp. 235-250, 2003.

[12] R. Langacker, Foundations of Cognitive Grammar, Volume I. Stanford, CA: Stanford University Press, 1987.

[13] C. J. Fillmore, "Valency issues in FrameNet," in Valency: Theoretical, Descriptive and Cognitive Issues, T. Herbst and K. Gotz-Votteler, Eds. Mouton de Gruyter, Berlin, 2007, pp. 129-160.

[14] J. Barwise, "Scenes and other situations," The Journal of Philosophy, vol. 78, no. 7, pp. 369-397, 1981.

[15] J. Barwise and J. Perry, Situations and Attitudes, MIT Press, Cambridge, MA, 1983.

[16] K. Devlin, "John Barwise's papers on natural language semantics," in The Bulletin of Symbolic Logic, vol. 10, no. 1, 2004.

[17] J. E. Fenstad, P.-K. Halvorsen, T. Langholm, and J. van Benthem, Situations, Language and Logic, Dordrecht: Reidel, 1987.

[18] G. Plotkin, "An illative theory of relations," in Situation Theory and Its Applications, R. Cooper, K. Mukai and J. Perry, eds. CSLI Lecture Notes Number 22: CSLI Publications, vol. 1, 1990.

[19] P. Aczel and R. Lunnon, "Universes and parameters," in Situation Theory and Its Applications, J. Barwise, J. M. Gawron, G. Plotkin, and S. Tutiya, Eds. CSLI Lecture Notes Number 26: CSLI Publications, 1991, vol. 2.

[20] R. Lunnon, "Generalized universes," Ph.D. thesis, University of Manchester, 1991.

[21] D. Davidson, "The logical form of action sentences," in The Logic of Decision and Action, N. Rescher, ed. Pittsburgh: University of Pittsburgh Press, 1967, pp. 81-94.

[22] R. Schäfer, "Arguments and adjuncts at the syntax-semantics interface," Ph.D. thesis, University of Göttingen, 2010.

[23] N. Chomsky, Syntactic Structures, The Hague: Mouton, 1957.

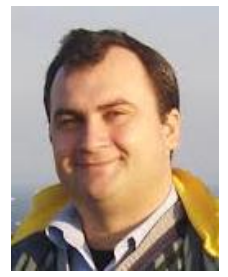

Cenk Atlig is an Asst. Professor at the Department of Computer Programming of Trakya University, Turkey. He is holder of number of international awards. He has authored several papers in international conference proceedings, and has been actively serving as a European Union project evaluator.

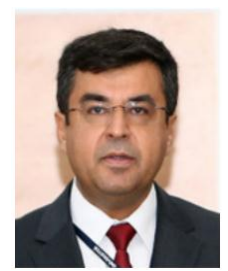

Yılmaz Kılıçaslan is a professor at the Department of Computer Engineering of Trakya University, Turkey. He has authored several papers in refereed journals and international conference proceedings, and has been actively serving as a reviewer for international journals and conferences.

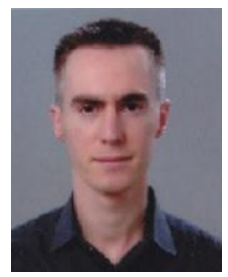

Gurkan Tuna is an Assoc. Professor at the Department of Computer Programming of Trakya University, Turkey (2006-). He has authored several papers in refereed journals and international conference proceedings, and has been actively serving as a reviewer for international journals and conferences. 